\section{Evaluación de la micro-deformación de la zona maxilar anterior con regeneración. Análisis de elementos finitos}

\section{Evaluation of anterior maxillar microstrain with bone regeneration. A finite element analysis}

\section{Resumen}

Objetivo. El objetivo de este estudio fue conocer y determinar la micro-deformación y distribución de los esfuerzos en el espesor de hueso maxilar anterior regenerado y rehabilitado con un implante usando el análisis de elementos finitos (MEF). Métodos. Se modeló un espesor del hueso maxilar con regeneración ósea de $1,5 \mathrm{~mm}$ por vestibular con un implante dental en posición de un incisivo central superior (hueso esponjoso, cortical, hueso regenerado, implante y componentes protésicos). Las variables incluidas en el modelado fueron el módulo de elasticidad, la razón de Poisson para todos los componentes. Se aplicó una carga de $200 \mathrm{~N}$ en dirección oblicua en la superficie palatina de la corona para calcular la distribución de los esfuerzos y la micro-deformación sobre el componente implante-hueso. Resultados. Los valores de máximo esfuerzo equivalente von Mises se encontraron en el hueso cortical $(44,89 \mathrm{MPa})$ que rodea el cuello del implante y se concentraron hasta las primeras cuatro roscas del implante adyacentes al hueso nativo en la zona palatina con hueso cortical mientras en el hueso regenerado en la zona vestibular se concentró hasta la rosca seis, disminuyendo los esfuerzos (2,5 MPa), y la microdeformación ósea fue mayor en el hueso cortical $(943 \mu \varepsilon)$ comparado con el hueso nativo (214 $\mu \varepsilon$ ). Conclusiones. La distribución de los esfuerzos y micro-deformación generados en el modelo se comportaron de manera diferente según el tipo de hueso (cortical, esponjoso, regenerado), donde el hueso cortical resiste los mayores esfuerzos y micro-deformaciones, distribuyendo menos carga al hueso regenerado.

Palabras clave: Análisis de elementos finitos; Módulo de elasticidad; Implantes dentales (fuente: DeCS BIREME).

\begin{abstract}
Objetive. The purpose of this study was to know and determine the microstrain and stress distribution in bucal bone, regenerated and rehabilitated with an implant using the finite element analysis (FEA). Methods. The thickness of maxillar bone modeling with bucal bone regeneration was $1.5 \mathrm{~mm}$ with a dental implant in position of the central incisor (Cancellous, cortical and regenerated bone, implant and prosthetic components).
\end{abstract}

\section{Artículo Original}

\author{
Johan Ivan Aguilar Henao ${ }^{1, a}$, Junes Abdul Villarraga Ossa ${ }^{1, b}$, \\ Federico Latorre Correa ${ }^{1, a}$ \\ ${ }^{1}$ Universidad de Antioquia, Facultad de Odontología. \\ Medellín, Colombia.

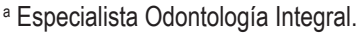 \\ ${ }^{\mathrm{b}}$ Magíster en Ingeniería Mecánica.

\section{Correspondencia:} \\ Federico Latorre Correa \\ Correo electrónico: latorre.federico29@gmail.com \\ Universidad de Antioquia. Facultad de Odontología. Calle \\ 64 N. ${ }^{\circ} 52-59$ Of. 107. Apartado Postal1226.

\section{Coautores:} \\ Johan Ivan Aguilar Henao \\ johan1021@hotmail.com \\ Junes Abdul Villarraga Ossa \\ junes.villarraga@udea.edu.co

\section{Editor:} \\ Yuri Castro-Rodríguez \\ Universidad Nacional Mayor de San Marcos, Perú.
}

Conflicto de intereses: no existe un posible conflicto de intereses en este manuscrito.

Fuente de financiamiento: Universidad de Antioquia.

\section{Recibido: $17 / 02 / 19$}

Aceptado: 05/04/19

Publicado: 05/06/19 
The variables were the modulus of elasticity and Poisson`s ratio for all the components in the model. A load of $200 \mathrm{~N}$ was applied to the palatal surface of the crown in order to calculate the microstrain stress distribution of the bone. Results. The maximum equivalent stress values of von Mises were found in the cortical bone (44.89 MPa) surrounding the neck of the implant and concentrated on the first four implant threads adjacent to the native bone in the palatal area, with cortical bone, while in the regenerated bone, in the vestibular area, it was concentrated until thread six, decreasing stress $(2.5 \mathrm{MPa})$, and bone microdeformation was greater in cortical bone $(943 \mu \varepsilon)$ compared to native bone $(214 \mu \varepsilon)$. Conclusions. The distribution of stresses and microstrain generated on the model, had different behavior depending on the type of bone (Cortical, cancellous, regenerated bone), the rigid cortical bone with bigger modulus of elasticity resisted more efforts and microstrain just like the implant that had a higher modulus of elasticiy and distributed less effort over the regenerated bone.

Keywords: Elastic modulus; Dental implants; Finite element analysis (source: $\mathrm{MeSH}$ NLM).

\section{Introducción}

Diversos factores ocasionan la pérdida de dientes en la zona anterior del maxilar, una alternativa de rehabilitación son los implantes oseointegrados, los cuales deben tener un mínimo espesor óseo para tener resultados estéticos predecibles ${ }^{1,2}$. En el momento que este espesor no se presenta, puede lograrse mediante la regeneración ósea ${ }^{3,4}$.

El espesor óseo en la zona anterior del maxilar, posterior a la pérdida dentaria, se afecta por la reabsorción de la tabla cortical externa, proceso progresivo, irreversible y crónico ${ }^{5}$. Luego de la pérdida de incisivos centrales superiores, la tabla ósea vestibular, a los 30 días, presenta una reabsorción del $30 \%{ }^{6-12}$. La disminución de hueso en la zona vestibular es más marcada que en la zona palatina debido al menor espesor óseo ${ }^{13-15}$. Botticelli et al. ${ }^{10}$ en el 2004, refirieron una reabsorción horizontal de la cresta ósea maxilar del $56 \%$ por vestibular, Braut et al. ${ }^{15}$ en el 2011 encontraron que en la parte anterior del hueso maxilar, una pared ósea delgada, menor a 1 mm, está presente en un 80,1\%. Huynh-Ba et al. ${ }^{16}$ en el 2010 demostraron que el $90 \%$ de las paredes óseas vestibulares en la zona estética tienen un grosor inferior a $1 \mathrm{~mm}$, Januario et al. ${ }^{17}$ en el 2011, reportaron que en el maxilar una pared ósea vestibular menor a $1 \mathrm{~mm}$ de espesor está presente en el $85 \%$ de los casos y el $50 \%$ tenían un espesor de tabla ósea menor de $0,5 \mathrm{~mm}$. En zonas anteriores de demanda estética, los parámetros de amplitud de la tabla cortical vestibular se consideran en $2 \mathrm{~mm}$, para que exista estabilidad del tejido blando, este parámetro se ha tomado basado en ideales sin evidencia científica ${ }^{18,19}$.

Posterior a la regeneración ósea se puede observar un hueso de baja calidad con características de hueso D3, en la clasificación de Misch ${ }^{20}$. El módulo de elasticidad del hueso tipo III es menor que el de tipo II y mayor que el de tipo IV, siendo el hueso tipo I diez veces más resistente a la compresión que el IV. La calidad y cantidad del hueso se han demostrado que influyen en las cargas alrededor de los implantes dentales ${ }^{20}$. Hueso esponjoso de baja densidad aumenta el esfuerzo en una mayor área del implante ${ }^{21}$. Estos esfuerzos producen micro-defor- maciones en las interfases óseas, debido a una menor resistencia del hueso comparado con el implante. En esta zona se presentan diferentes niveles de micro-deformación, los cuales van desde una disminución de la densidad ósea hasta producir grietas en el hueso, y dicha deformación se mide con una variable física llamada microstrain ${ }^{20-22}$.

El propósito de esta investigación fue evaluar la micro-deformación y el comportamiento de los esfuerzos en el espesor óseo del hueso regenerado vestibular con un implante. Se analizó la zona anterior del maxilar, la cual presenta una alta exigencia estética, la evaluación del hueso regenerado de la zona vestibular indicó el comportamiento mecánico de este y la posibilidad de la estabilidad en los tejidos blandos.

\section{Métodos}

El método de elementos finitos se utilizó para evaluar el comportamiento mecánico de hueso regenerado con una restauración implanto-soportada. Se realizó un modelo tridimensional del espesor óseo de hueso regenerado vestibular de $1,5 \mathrm{~mm}$, medida tomada de una serie de cortes tomográficos de hueso regenerado en la práctica privada, con un implante de diámetro $3,7 \mathrm{~mm}$ y $13 \mathrm{~mm}$ de longitud, pilar, tornillo, cofia cerámica, corona cerámica, hueso cortical palatino, hueso esponjoso y precarga con las siguientes características: implante Zimmer ${ }^{\circ}$ para diente anterior incisivo central superior: $13 \mathrm{~mm}$ de longitud, $3,7 \mathrm{~mm}$ de diámetro, $3,5 \mathrm{~mm}$ de diámetro de plataforma, superficie MTX rugosa, hexágono interno 2,5 mm. Tapered Screw-Vent Implants TSVB13. Fabricante: Zimmer ${ }^{\circ}$ Dental 1900 Aston Avenue Carlsbad, CA 92008-7308 USA. Referencia número: TSVB13. Casa Fabricante: Zimmer ${ }^{\circ}$ Dental1900 Aston Avenue Carlsbad, CA 92008-7308 USA. Longitud cervical 2,5 $\mathrm{mm}$, tercio medio $7,5 \mathrm{~mm}$, apical 3,0 $\mathrm{mm}$, distancia del paso de rosca $1,8 \mathrm{~mm}$, profundidad de la rosca 0,375 $\mathrm{mm}$, tipo de rosca triple.

Pilar de Zirconia Zimmer ${ }^{\bullet}$ con las siguientes características: precontorneado, recto, conexión interna hexagonal, diámetro de $3,5 \mathrm{~mm}$, perfil de emergencia de 4,5 $\mathrm{mm}$, altura del margen vestibular $1,0 \mathrm{~mm}$, anillo de 
titanio en la interfase con el implante. Referencia número: ZRA341S. Casa Fabricante: Zimmer ${ }^{\oplus}$ Dental1900 Aston Avenue Carlsbad, CA 92008-7308 USA. Cofia cerámica en disilicato de litio, con recubrimiento cerámico con E-max-Ceram.

El hueso incluyó cortical palatina y esponjoso tipo D3, descrito por Misch ${ }^{20}$, el tipo D3 presenta un hueso trabecular fino, fue modelado a partir de un corte sagital del maxilar, incluyendo el hueso alveolar regenerado y cortical palatina. Dadas las condiciones del modelado se asumió una oseointegración del 100\% (Figura 1).

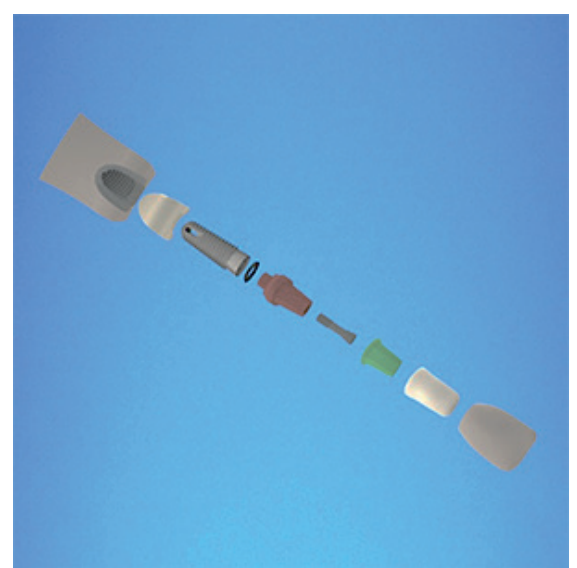

Figura 1. Imagen renderizada de los componentes del modelo

Se asignaron un conjunto de propiedades elásticas al modelo del hueso. Lo ideal es que estas propiedades presentaran anisotropía. Considerando que el modulo elástico del hueso cortical en dirección vestíbulo-palatino e ínfero-superior no son diferentes y los de un hueso esponjoso en dirección vestíbulo-palatino y mesiodistal son similares, se propuso el hueso como transversalmente isotrópico ${ }^{23,24}$. Con estas consideraciones se evaluaron cinco propiedades elásticas en lugar de nueve para un material ortotrópico. Esta aproximación de isotropía transversal representa mejor la realidad de la anisotropía del hueso ${ }^{24}$.

Para el modelo geométrico tridimensional, se utilizó el software CAD de SolidWorks (SolidWorks, SolidWorks Corp, Concord, MA, USA). Para obtener la deformación, el esfuerzo y el desplazamiento, mediante el modelo, se utilizaron las constantes elásticas: modelo de Young y coeficiente de Poisson, para cada uno de los elementos que se analizaron (Tabla 1).

La carga aplicada fue de $200 \mathrm{~N}$, que correspondió a la carga oclusal promedio reportada por la literatura para el sector anterior ${ }^{33}$. La micro-deformación se evaluó de forma directamente proporcional a la deformación, de forma que un $\mu \varepsilon$ equivale a $10^{6} \mathrm{~m} / \mathrm{m}$.

Una vez se elaboró el diseńado y modelado, se exportaron al software de elementos finitos llamado ANSYS para realizar el análisis del comportamiento mecánico de la estructura. Se realizó un análisis elástico lineal estático donde se utilizaron elementos tetraédricos, con el fin de obtener una mejor aproximación de las geometrías de las partes, de esta manera se obtuvo una malla tridimensional de elementos finitos de los componentes que conforman el modelo (Figura 2). Los resultados se presentan de manera gráfica con intensidad de colores que definen la magnitud de los esfuerzos principales en cada una de las superficies descritas.

Tabla 1. Propiedades mecánicas de las estructuras y materiales modelados

\begin{tabular}{|c|c|c|c|c|c|c|c|}
\hline \multirow[t]{2}{*}{ Material } & \multicolumn{3}{|c|}{$\begin{array}{l}\text { Limite elástico } \\
\mathrm{E}(\mathrm{MPa})\end{array}$} & \multicolumn{3}{|c|}{$\begin{array}{l}\text { Coeficiente de } \\
\text { Poisson - V }\end{array}$} & \multirow[t]{2}{*}{$\begin{array}{l}\text { Límite de } \\
\text { fluencia } \\
\text { (Mpa) }\end{array}$} \\
\hline & $x$ & Y & $\mathrm{Z}$ & YZ & $X Y$ & $X Z$ & \\
\hline Hueso cortical ${ }^{24}$ & 12,600 & 12,600 & 19,400 & 0,253 & 0,3 & 0,253 & $60-120$ \\
\hline Hueso regenerado ${ }^{24}$ & 230 & 42 & 230 & 0,01 & 0,05 & 0,32 & $60-120$ \\
\hline Hueso esponjoso ${ }^{24}$ & 1,148 & 210 & 1,148 & 0,50 & 0,32 & 0,10 & $60-120$ \\
\hline Encía $^{25}$ & 19,6 & 19,6 & 19,6 & 0,30 & 0,30 & 0,30 & N/A \\
\hline Implante de titanio 25,26 & 110.000 & 110.000 & 110.000 & 0,33 & 0,33 & 0,33 & 800 \\
\hline Tornillo del pilar titanio 25,26 & 110.000 & 110.000 & 110.000 & 0,33 & 0,33 & 0,33 & 800 \\
\hline Pilar de Circona ${ }^{27,28}$ & 200.000 & 200.000 & 200.000 & 0,27 & 0,27 & 0,27 & 900 \\
\hline $\begin{array}{l}\text { Cemento resinoso Rely } X^{\mathrm{TM}} \text { Unicem } \\
2 \text { Automix }{ }^{29}\end{array}$ & 6.600 & 6.600 & 6.600 & 0,300 & 0,300 & 0,300 & 48 \\
\hline $\begin{array}{l}\text { Corona Cerámica Feldespática IPS } \\
\text { e.max Ceram (IvoclarVivadent) }\end{array}$ & 69.000 & 69.000 & 69.000 & 0,280 & 0,280 & 0,280 & 90 \\
\hline $\begin{array}{l}\text { Estructura cerámica en disilicato de } \\
\text { litio (IPS e Max press) }{ }^{32}\end{array}$ & 95.000 & 95.000 & 95.000 & 0,230 & 0,230 & 0,230 & 350 \\
\hline
\end{tabular}




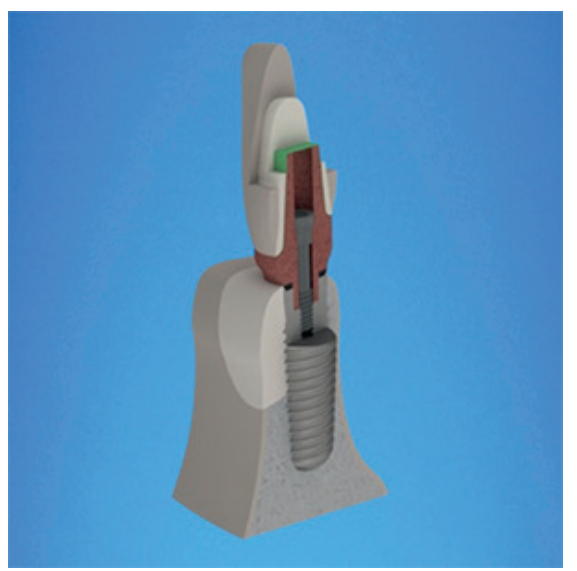

Figura 2. Corte sagital con los componentes del modelo

Tabla 2. Esfuerzo von Mises y micro-deformación posterior a la aplicación de la carga estática

\begin{tabular}{lcc}
\hline \multicolumn{1}{c}{ Material } & $\begin{array}{c}\text { Esfuerzo von Mises } \\
(\mathrm{MPa})\end{array}$ & $\begin{array}{c}\text { Micro-deformación } \\
(\mu \varepsilon)\end{array}$ \\
\hline Implante de titanio & 210,33 & NA \\
Hueso esponjoso & 7,02 & 597 \\
Hueso cortical & 44,89 & 943 \\
Hueso regenerado & 2,5 & 214 \\
\hline
\end{tabular}

\section{Resultados}

El análisis de elementos finitos se realizó para la carga aplicada de $200 \mathrm{~N}$ en los elementos modelados. Esta evaluación permitió conocer los efectos generados en todo el componente hueso-implante, nativo y regenerado debido a la carga aplicada. Estos efectos son: los valores de micro-deformación y la distribución de los esfuerzos equivalentes de von Mises (Tabla 2).

Distribución de esfuerzo en el implante. El máximo equivalente von Mises se localizó en vestibular y palatino del área cervical del implante, y en la primera rosca interna. El máximo esfuerzo von Mises para el modelo en carga estática fue de 210,33 MPa. Valor inferior al límite elástico del titanio. El esfuerzo compresivo fue de $212 \mathrm{MPa}$, concentrándose en el área vestibular.

Distribución de esfuerzo en el hueso peri-implante. Los valores de máximo esfuerzo equivalente von Mises se encontraron en el hueso que rodea el cuello del implante. No se presentó esfuerzo en el hueso más apical. Estos se concentraron hasta las primeras cuatro roscas del implante adyacentes al hueso nativo en la zona palatina con hueso cortical. En contraste el hueso regenerado en la zona vestibular las concentró hasta la rosca seis, aumentando los esfuerzos en una mayor área, comparado con el hueso nativo. El máximo equivalente von Mises para el hueso regenerado se localiza en la unión de este con el hueso nativo, decreciendo hacia la zona externa del modelo.

Distribución de micro-deformación en el hueso peri-implantar. Los valores máximos de micro-deformación en el hueso alrededor del implante se muestran en la Tabla 2. El valor máximo estaba por debajo de 944 $\mu \varepsilon$. Estos se presentaron en la parte más cervical del hueso, alrededor del implante. Mostrando mayor concentración en la zona palatina de hueso cortical, adyacente a las tres primeras roscas, cuando es comparado con el hueso esponjoso y regenerado.

\section{Discusión}

La regeneración ósea en la zona anterior del maxilar es un procedimiento común, aunque falta evidencia sobre las propiedades biológicas, mecánicas y la resistencia final de este tipo de hueso. Actualmente hay dificultades para evaluar la biomecánica y comportamiento del hueso regenerado, debido a problemas éticos, por costos y todas las dificultades de los estudios clínicos, por lo que existe poco seguimiento reportado. Por esto, herramientas de análisis como el método de elementos finitos son importantes para su evaluación. Este estudio, es una aproximación a la situación clínica, para tal efecto fue primordial el modelado de la geometría del implante, componentes protésicos, el espacio para el implante, calidad de hueso regenerado y nativo.

Los esfuerzos y la micro-deformación obtenidos en este estudio presentaron un comportamiento muy diferente en el hueso cortical, trabecular y regenerado. Según lo expuesto por Isidor et al. ${ }^{34}$ en el 2006 y Sevimay et al. ${ }^{35}$ en el 2005, el comportamiento de los esfuerzos depende de las características del hueso que los recibe. La mayor parte de los esfuerzos en el hueso se concentraron en la cortical palatina en contacto con el implante en la porción más cervical, estos fueron $42,39 \%$ mayor que el registrado en el hueso regenerado vestibular, lo que 
coincide en cierta medida (el modelo de este estudio no presento cortical vestibular) con lo publicado por Sevimay et al. ${ }^{35}$ en el 2005, por Kitamura et al. ${ }^{36}$ en el 2004, y Danza et al. ${ }^{37}$ en el 2009, quienes relatan que la concentración de los esfuerzos en esta zona, se debe a la mayor resistencia a la deformación de la cortical con respecto al hueso esponjoso. Este último presento los máximos esfuerzos von Mises en la zona cervical disipándolos en una zona más amplia al compararlo con el cortical.

En este estudio los valores máximos de micro-deformación estuvieron por debajo de $944 \mu \varepsilon$. concentrándose en la zona palatina de hueso cortical, siendo un $72,9 \%$ superior a lo observado en zona vestibular con hueso regenerado y $34,6 \%$ mayor al registrado en hueso esponjoso. En 1987, Frost afirmó que las distintas presiones ejercidas sobre el tejido óseo provocan una deformación elástica del mismo. Para comprobar esta afirmación, Frost utilizó la variable física microstrain, la cual se genera como producto de las cargas que recibe el hueso en relación con el volumen óseo inicial y el volumen óseo final ${ }^{38}$. De acuerdo con esto Frost ${ }^{39-42}$ describió unos intervalos de micro-deformación, donde de 0 a $200 \mu \varepsilon$ existe una disminución de la densidad ósea, de 200 a $1000 \mu \varepsilon$ la remodelación ósea está en equilibrio, de 1 000 a $3000 \mu \varepsilon$ se estimula la actividad de remodelación, de 3000 a $10000 \mu \varepsilon$ hay sobrecarga patológica, donde se generan fracturas por fatiga y reabsorción. Por último, se encuentra una zona de fractura espontánea, con deformación entre el 1 y $2 \%$, que equivale a 10 000 y $20000 \mu \varepsilon^{22}$. Según lo reportado por este autor, todos los valores de micro-deformación experimentados por el hueso de nuestra investigación, estarían dentro de los límites de adaptación, siendo el hueso cortical de la zona palatina el que presenta los valores más altos de micro-deformación.

Se considera que las técnicas de regeneración ósea constituyen un factor de riesgo para el éxito del tratamiento con implantes en la zona anterior del maxilar, por su inserción en un tejido formado por hueso y un biomaterial injertado, sin embargo, múltiples estudios clínicos y radiográficos muestran alta tasa de éxito con estas técnicas, presentando resultados superiores al $95 \%{ }^{43-48}$. Hay pocos estudios en cantidad y calidad que evalúen el comportamiento biomecánico de implantes en hueso regenerado, por lo cual se hace imposible contrastar nuestros resultados con otras investigaciones similares. No obstante, nuestros datos están de acuerdo con los resultados de los estudios clínicos y radiográficos que muestran que la utilización de hueso regenerado en la zona anterior del maxilar es un tratamiento confiable en ciertas condiciones clínicas.

En este estudio se observó que al momento de soportar y distribuir las cargas alrededor del implante, el hueso regenerado participó en menor medida que los demás componentes. Se le adjudican propiedades de un material de relleno, más que de soporte. De los resultados se puede inferir que la estabilidad del hueso y tejidos alrededor del implante se dan a expensas del hueso nativo presente, principalmente por la cortical palatina que recibe la mayor parte de los esfuerzos y micro-deformación generadas en esta simulación. Es importante tener precaución al momento de implementar el tratamiento con implantes y grandes superficies de hueso regenerado, teniendo en cuenta que la presencia de hueso regenerado en este estudio aumentó los esfuerzos en un área mayor del implante, lo que concuerda con lo encontrado por Sevimay et al. ${ }^{35}$ en el 2005 al evaluar los esfuerzos en huesos con diferentes densidades, donde observaron que los de peor calidad, como el hueso regenerado presentaban mayores esfuerzos con una dispersión más apical.

En estudios en la región posterior como el de Loyola-Gonzáles et al. ${ }^{49}$ encontraron poco o reducido esfuerzo sobre el hueso regenerado debido a sus propiedades mecánicas y a estar distante de la zona de mayores esfuerzos y que elementos con mayor módulo de elasticidad concentran los mayores valores, resultados que están de acuerdo con los presentados en esta investigación.

Esta investigación concuerda con la revisión sistemática y metanálisis de Salvi et al. ${ }^{50}$ en el 2018, donde no encuentran diferencias significativas entre hueso nativo y hueso regenerado, sin embargo observan mayor variabilidad y menos predecibilidad en el hueso regenerado. Dentro de las limitaciones de este tipo de estudio, donde se hace un análisis de los esfuerzos en el sector anterior, el cual ha sido analizado en varios estudios pero no se evaluó el hueso regenerado, debido a las dificultades con su observación y comprendiendo que es una simulación matemática, la cual es una información que no se puede considerar como evidencia clínica, se podrían dar las siguientes conclusiones: la estabilidad del hueso y el tejido alrededor del implante evaluado en esta investigación se da a expensas del hueso nativo, principalmente por la cortical palatina que resiste la mayor parte del esfuerzo y la micro-deformación en el modelo. La presencia de hueso regenerado en esta simulación aporta poco soporte biomecánico al modelo aumentando los esfuerzos en un área mayor alrededor del implante.

\section{Referencias bibliográficas}

1. Petersen PE. The World Oral Health Report 2003: continuous improvement of oral health in the 21 st century - the approach of the WHO Global Oral Health Programme. Community Dent Oral Epidemiol. 2003;31(1):3-23.

2. Raigrodski AJ, Chiche GJ. The safety and efficacy of anterior ceramic fixed partial dentures: A review of the literature. J Prosthet Dent. 2001;86(5):520-5.

3. Esposito M, Grusovin M, Coulthard P, Worthington H. The Efficacy of Various Bone Augmentation Procedures for Dental Implants: A Cochrane Systematic Review of Randomized Controlled Clinical Trials. Int J Oral \& Maxillofac Implants. 2006;21(5):696-710.

4. Jensen S, Terheyden H. Bone Augmentation Procedures in Localized Defects in the Alveolar Ridge: Clinical Results with Different Bone Grafts and Bone-Substitute Materials. Int J Oral \& Maxillofac Implants. 2009;2:218-236. 
5. Wang HL, Kiyonobu K, Neiva RF. Socket augmentation: rationale and technique. Implant Dent. 2004;13(4):28696.

6. Araújo M, Lindhe J. Dimensional ridge alterations following tooth extraction. An experimental study in the dog. J Clin Periodontol. 2005;32(2):212-8.

7. Araújo M, Linder E, Wennström J, Lindhe J. The influence of Bio-Oss Collagen on healing of an extraction socket: an experimental study in the dog. Int J Periodontics Restorative Dent. 2008;28(2):123-35.

8. Nevins M, Stein J. The Placement of Maxillary Anterior Implants. In: Nevins M, Mellonig J. Implant therapy: Clinical Approaches and Evidence of Success. Volume 2. Carol Stream, Illinois: Quintessence Publishing Co, Inc; 1998. p.p $111-127$.

9. Ding X, Liao SH, Zhu XH, Zhang XH, Zhang L. Effect of diameter and length on stress distribution of the alveolar crest around immediate loading implants. Clin Implant Dent Relat Res. 2009;11(4):279-87.

10. Botticelli D, Berglundh T, Lindhe J. Hard-tissue alterations following immediate implant placement in extraction sites. J Clin Periodontol. 2004;31(10):820-8

11. Caubet J, Heras I, Sánchez J, Morey M, Iriarte J. Management of anteroposterior bone defects in aesthetic restoration of the front teeth. Rev Esp Cirug Oral y Maxilofac. 2009;31(2):81-97.

12. Bozkaya D, Muftu S, Muftu A. Evaluation of load transfer characteristics of five different implants in compact bone at different load levels by finite elements analysis. J Prosthet Dent. 2004;92(6):523-30.

13. Araújo MG, Sukekava F, Wennström JL, Lindhe J. Ridge alterations following implant placement in fresh extraction sockets: an experimental study in the dog. J Clin Periodontol. 2005;32(6):645-52.

14. Tomasi C, Sanz M, Cecchinato D, Pjetursson B.Bone dimensional variations at implants placed in fresh extraction sockets: a multilevel multivariate analysis. Clin Oral Implants Res. 2010;21(1):30-6.

15. Braut V, Bornstein MM, Belser U, Buser D. Thickness of the anterior maxillary facial bone wall- a retrospective radiographic study using cone beam computed tomography. Int J Periodontics Restorative Dent. 2011;31(2):125-31

16. Huynh-Ba G, Pjetursson B, Sanz M, Cecchinato D, Ferrus J, Lang N. Analysis of the socket bone wall dimensions in the upper maxilla in relation to immediate implant placement. Clin Oral Implants Res. 2010;21(1):37-42.

17. Januário AL, Duarte WR, Barriviera M, Mesti JC, Araújo MG, Lindhe J. Dimension of the facial bone wall in the anterior maxilla: a cone-beam computed tomography study. Clin Oral Implants Res. 2011;22(10):1168-71.

18. Spray RJ, Black GC, Morris HF. The influence of bone thickness on facial marginal bone response: stage 1 placement through stage 2 uncovering. Ann Periodontol 2000;5:119-28.

19. Elian N, Ehrlich B, Jalbout ZN, Tarnow DP. Advanced Concepts in Implant Dentistry: Creating the "Aesthetic Site Foundation". Dent Clin N Am. 2007; 51:547-63.
20. Misch C. Bone density: A key determinant for treatment planning. Part I: diagnosis and rationale. In: Contemporary Implant Dentistry. $3^{a}$ ed. Ed Mosby, Elsevier, Canada 2009. p. 130-140.

21. Petrie C, Williams J. Comparative evaluation of implant designs: influence of diameter, length, and taper on strains in the alveolar crest. Clin Oral Implants Res. 2005;16(4):486-494.

22. Chou HY, Müftü S, Bozkaya D. Combined effects of implant insertion depth and alveolar bone quality on periimplant bone strain induced by a wide-diameter, short implant and a narrow-diameter, long implant. J Prosthet Dent. 2010;104(5):293-300.

23. Ding X, Liao SH, Zhu XH, Zhang XH, Zhang L. Effect of diameter and length on stress distribution of the alveolar crest around immediate loading implants. Clin Implant Dent Relat Res. 2009;11(4):279-87.

24. Kurniawan D. Finite element analysis of bone-implant biomechanics: refinement throughfeaturing various osseointegration conditions. Int J Oral Maxillofac Surg. 2012;41(9):1090-1096.

25. Kayabaşı O, Yüzbasıoğlu E, Erzincanlı F. Static, dynamic and fatigue behaviors of dental implant using finite element method. Adv Eng Softw. 2006;37(10):649-58.

26. Niinomi M. Mechanical properties of biomedical titanium alloys. Mater Sci Eng. 1998;243(1-2):231-6.

27. Papanagiotou HP, Morgano SM, Giordano RA, Pober R. In vitro evaluation of low-temperature aging effects and finishing procedures on the flexural strength and structural stability of Y-TZP dental ceramics. J Prosthet Dent. 2006;96(3):154-64

28. Luthardt RG, Holzhüter MS, Rudolph H, Herold V, Walter MH.CAD/CAM-machining effects on Y-TZP zirconia. Dent Mater. 2004;20(7):655-62.

29. RelyXUnicem 2 Automix - Technical Data Sheet [Internet]. [citado 2013 may 19]. Available a partir de http://multimedia.3m.com/mws/mediawebserver?mwsId=66666UF6EVsSyXTtnxf218TXEVtQEVs6EVs6EVs6E666666--\&fn=rx_u2_auto_tds.pdf

30. IPS e.maxCeramS cientific Documentation [Internet]. [citado 2013 may 19]. Available a partir de: http://www. infinident.com/ecomaXL/get_blob.php?name=IPS_e. max_Ceram_Scientific_Doc.pdf

31. Ozkurt Z, Kazazoglu E, Unal A. In vitro evaluation of shear bond strength of veneering ceramics to zirconia. Dent Mater J. 2010;29(2):138-46.

32. IPS e.max Press. Scientific Documentation [Internet]. [Citado 2013 may 19]. Available a partir de: http:// www.ivoclarvivadent.com/zoolu-website/media/document/9808/IPS+e-max+Press

33. Morneburg TR, Proschel PA. In vivo forces on implants influenced by occlusal scheme and food consistency. Int J Prosthodont. 2003;16(5):481-6.

34. Isidor F. Influence of forces on peri-implant bone. Clin Oral Implants Res. 2006;17:8-18.

35. Sevimay M, Turhan F, Kiliçarslan M, Eskitascioglu G. Three-dimensional finite element analysis of 
the effect of different bone quality on stress distribution in an implant-supported crown. J Prosthet Dent. 2005;93(3):227-234.

36. Kitamura E, Stegaroiu R, Nomura S, Miyakawa O. Biomechanical aspects of marginal bone resorption around osseointegrated implants: considerations based on a three-dimensional finite element analysis. Clin Oral Implants Res. 2004;15(4):401-412.

37. Danza M, Palmieri A, Farinella F, Brunelli G, Carinci F, Girardi A, Spinelli G, et al Three dimensional finite element analysis to detect stress distribution in spiral implants and surrounding bone. Dent Res J. 2009;6( 2):59-64.

38. Frost HM. Bone "mass" and the "mechanostat": a proposal. Anat Rec. 1987;219(1):1-9.

39. Frost HM. From Wolff's law to the Utah paradigm: insights about bone physiology and its clinical applications. Anat Rec. 2001;262(4):398-419.

40. Frost HM. Perspectives: a proposed general model of the "mechanostat" (suggestions from a new skeletal-biologic paradigm). Anat Rec. 1996; 244(3):139-147.

41. Frost HM. Skeletal structural adaptations to mechanical usage (SATMU): 2. Redefining Wolff's law: the remodeling problem. Anat Rec. 1990;226(4):414-422.

42. Frost HM. Wolff's Law and bone's structural adaptations to mechanical usage: an overview for clinicians. Angle Orthod. 1994;64(4):187-212.

43. Donos N, Mardas N, Chadha V. Clinical outcomes of implants following lateral bone augmentation: systematic assessment of available options (barrier membranes, bone grafts, split osteotomy). J Clin Perio. 2008;35(8):173-202.
44. Chiapasco M, Zaniboni M, Boisco M. Augmentation procedures for the rehabilitation of deficient edentulous ridges with oral implants. Clin Oral Implants Res. 2006;17(2):136-159.

45. Fu J, Oh T, Benavides E, Rudek I, Wang H. A randomized clinical trial evaluating the efficacy of the sandwich bone augmentation technique in increasing buccal bone thickness during implant placement surgery: I. Clinical and radiographic parameters. Clin Oral Implants Res. 2014;25(4):458-467.

46. Kan J, Rungcharassaeng K, Sclar A, Lozada J. Effects of the facial osseous defect morphology on gingival dynamics after immediate tooth replacement and guided bone regeneration: 1-year results. J Oral Maxillofac Surg. 2007;65(7):13-19.

47. Kuchler U, von Arx T. Horizontal ridge augmentation in conjunction with or prior to implant placement in the anterior maxilla: a systematic review. Int J Oral Maxillofac Implants. 2014;29(1):14-24.

48. Cosyn J, Eghbali A, Hanselaer L, De Rouck T, Wyn I, De Bruyn H, et al. Four modalities of single implant treatment in the anterior maxilla: a clinical, radiographic, and aesthetic evaluation. Clin implant Dent Relat Res 2013;15(4):517-530.

49. Loyola-Gonzáles PO,Torass D, Dominguez A. Estudio comparativo sobre el comportamiento y la dsitribución de las tensiones en implantes dentales cortos e implantes dentales estándares en la región posterior del maxilar superior. Un estudio en elementos finitos. Rev clin periodoncia implantol rehabil oral. 2016;9(1):36-41

50. Salvi GE, Monje A, Tomasi C. Long-term biological complications of dental implants placed eiher or in augmented sites: A systematic review and meta-analysis. Clin Oral Impl Res. 2018;29(16):294-310. 
